\title{
2020 Fiscal Year Contributors
}

$\mathrm{T}$ his list celebrates the generosity of donors to APSA. We are honored to recognize those who made a donation to support our work during the 2019-20 fiscal year (October 1, 2019-September 30, 2020). Thank you for helping us continue critical programs and professional resources for APSA members around the world.

Gabriele Abels Scott F. Abernathy Alan I. Abramowitz Martha A. Ackelsberg Tony Affigne

Amel F. Ahmed

\section{Geoff Allen}

Eugene J. Alpert

Bruce E. Altschuler

Stephen Amberg

Patricia Amberg-Blyskal

J. Theodore Anagnoson Jason Anastasopoulos

Ashley Anderson

Connie Anthony

Reiko Arami

Lara Arnold

Joseph Asunka

John Attanasio

Scott Barclay

Elizabeth Barringer

Harold F. Bass

Regina A. Bateson

Angie Maritza BautistaChavez

Kristy A. Belton

Seyla Benhabib

Seth B. Benjamin

Jonathan Benjamin-

Alvarado

Stanley P. Berard

Adam Bernbaum

Terri Bimes

Kelvin Calhoun Black

Graeme Blair

Charles H. Blake

Brian Blankenship

Janice L. Bockmeyer

Charles R. Boehmer

Alison Boehmer

Catherine Boone

W. James Booth

Elizabeth T. Boris

Matthew H. Bosworth
Carew E. Boulding

Rachel E. Bowen

Daniel Bowen

Ann O'M. Bowman

Daniel Bruce Braaten

Jonathan Doc Bradley

James M. Brasfield

Matthew James Brazil

Sheryl Demorest Breen

Christine Thurlow Brenner

Marijke Breuning

Michael A. Brintnall

Mitchell Brown

Colin M. Brown

Heath Brown

Stanley C. Brubaker

Adeyinka Ayobambo

Bruce Omotunde

Claudia Franziska

Bruehwiler

Haeusermann

Kathleen M. Bruhn

Anthony R. Brunello

Jeremy Buchman

Cynthia Burack

John Francis Burke

Nancy Burns

Charles E. Butterworth

Joseph Cammarano

Joel R. Campbell

Michael P. Canning

Devon Kyla Cantwell

Carnegie Corporation of

New York

Peter Carroll

Austin Carson

Niambi M. Carter

Kierstan Marie Carter

Leo E. Casey

Gretchen G. Casper

Rutger Ceballos

Simone Chambers

Terrence Chapman

Nick Cheesman
Jonathan Joseph Chiarella Barbara Ann Chotiner

Beverly A. Cigler

Danielle P. Clealand

Ajenai S. Clemmons

Jean Clipperton

Cary Coglianese

Dara Kay Cohen

Lanelle Coleman

Meredith Conroy

Alexander Coppock

J. Kevin Corder

Cristina Corduneanu-Huci

Bryce Corrigan

Renee A. Cramer

Michael C. Craw

Neta C. Crawford

Vanessa Cruz Nichols

Paul J. Culhane

Michael Stuart Cummings

James E. Curtis, Jr.

Alexis Cuttance

Jennifer Marie Cyr

Rafaela Dancygier

Paolo Dardanelli

Daniel de Kadt

Valerie de Koeijer

Eline A. de Rooii

Adam de Sola Pool

Rebecca E. Deen

Christopher J. Deering

Richard E. DeLeon

Democracy Fund

Destination DC

Valerie D'Erman

Christine Di Stefano

Brendan J. Doherty

Julie Dolan

Jan Matti Dollbaum

Michael J. Donnelly

Cassy Dorff

Conor M. Dowling

Alex Dyzenhaus

Bianca Easterly

John Echeverri-Gent

Ruth M. Ediger

Sean D. Ehrlich

Barbara Elias

Antje Ellermann

Andrew Emerson

Ben Epstein

Kenneth Paul Erickson
Alfred B. Evans Jr.

Victoria A. Farrar-Myers

Henry Farrell

Michael K. Fauntroy

Jeffrey Feldman

Botond Feledy

John Ferejohn

Bonnie N. Field

Evgeny Finkel

Martha Finnemore

Richard S. Flickinger

Lynne E. Ford

Margaret J. Foster

Jane E. Fountain

Josue Alejandro Franco

David M. Frankford

Sekou Franklin

Clifford Frasier

Jeffry A. Frieden

Jason David Frye-Kolarik

Beth C. Fuchs

Carey and Josephine Fujii

Jeff Fujii

Haruhiro Fukui

Sean Gabaree

Fritz Gaenslen

Sean Gailmard

Deborah Galimberti

J. Andres Gannon

Marshall Ganz

John Armando Garcia

Marcela García-

\section{Castañon}

Kent Gardner

Jake Garringer

Alice L. George

Irwin N. Gertzog

Anthony "Jack" Gierzynski

Andra Gillespie

Terry Gilmour

Jane R. Gingrich

Aarie Glas

Sona N. Golder

Dariusz Gora

Lilly J. Goren

Kristin Goss

Charles W. Gossett

Doris A. Graber Trust

Joan Grace

Keneshia N. Grant

Scott L. Greer

Bernard N. Grofman
Colleen M. Grogan

Jeff Gulati

Kate Habegger

Henry E. Hale

Kathleen Hale

Michael G. Hanchard

Antoinette Handley

John W. Harbeson

Brian M. Harward

Mai Omer Hassan

Virginia Haufler

Jennifer P. Haydel

Morgan L. W. Hazelton

Jeffrey R. Henig

April A Herlevi

Margaret G. Hermann

Rodney E. Hero

William and Flora Hewlett

Foundation

Ronald Salem Hikel

John F. Hoadley

Bai Linh Hoang

William C. Hoffman

Marie Hojnacki

Alisha Caroline Holland

Barbara O. Holmes

Liesbet Hooghe

Daniel J. Hopkins

Jennifer Hopper

Yusaku Horiuchi

Sarah Loretto Houser

Marketa Houskova

Yasheng Huang

Juan Carlos Huerta

Sara Hughes

Karen M. Hult

Andy Humm

Vincent L. Hutchings

Susan D. Hyde

Nahomi Ichino

Maria de la Luz Inclan

Maxine Isaacs

John Ishiyama

Ivywood Foundation

Alan M. Jacobs

Gary C. Jacobson

Nakissa Puneh Jahanbani

Amaney Jamal

Sarah Elizabeth James

Alix J. Jansen

Jane S. Jaquette

Holly Jarman 
Maiah Jaskoski

James Jennings

Martha C. Johnson

Richard Johnston

Seth Jolly

Bryan D. Jones

Jyl J. Josephson

Anil Kalhan

Alice Kang

Elsa Babette Kania

Nick Kapoor

Yuri Kasahara

Nancy Kassop

Kenneth Kato

Peter J. Katzenstein

Ewing Marion Kauffman

Foundation

Joyce P. Kaufman

Christopher Kelaher

Ann C. Keller

Mark Kesselman

Christina Kiel

Drew Holland Kinney

Kathleen Klaus

Hidetaka Kobayashi

Robin A. Kolodny

Sina Jo Kramer

Michael Alan Krasner

Henry Krisch

Mona Lena Krook

George W S. Kuhn III

Andrew Kydd

Chryl Laird

Loren B. Landau

Bruce A. Larson

Regina G. Lawrence

David Lazer

Loan K. Le

David L. Leal

Sharon F. Lean

Matthew Lebo

Adam Lebovitz

Carol Skalnik Leff

Debra Lynn Leiter

Martin Adam Lessem

Benjamin Lessing

Margaret Levi

Jacob S. Lewis

Rose Lewis

Michael S. Lewis-Beck

Rachel Lienesch

Larry Lee Light

Staffan I. Lindberg

Jinee Lokaneeta

Burdett A. Loomis

Peter L. Lorentzen

Nancy Sue Love
David Lublin

Henry Luce Foundation

DeWayne L. Lucas

Monique L. Lyle

Ruofan Ma

Lauren M. MacLean

Raul L. Madrid

Cherie Maestas

Paasha Mahdavi

James Mahoney

Samantha Ann Majic

Aditi Malik

Carrie Manning

Greg Marfleet

Suzanne M. Marilley

Patchen Markell

Irving Leonard Markovitz

Renee Marlin-Bennett

Sherry L. Martin

Melissa Martinez

Valerie J. Martinez-Ebers

Donald J. Matthewson

Sarah Maxey

Lindsay Rose Mayka

Michael W. McCann

James A. McCann

Susan K. McCarthy

Alison Rios Millett

McCartney

Paula D. McClain

John P. McCormick

Karen M. McCurdy

Andrew S. McFarland

Mary A. McHugh

Benjamin McKean

John McMahon

Susan McWilliams

Nancy McWilliams

Kimberly Mealy

Brian Mealy

Taneisha Nicole Means

Elizabeth Meehan

Ken Meier

Tali Mendelberg

Matthew Mendez

David Menefee-Libey

Arthur E. Menna

Carol A. Mershon

Suzanne Mettler

Tamara Metz

John Michael Meyer

Melissa R. Michelson

Robert Mickey

Nicholas R. Miller

Aidan Milliff

Michael Minkenberg

Charles L. Mitchell
Cecilia Hyunjung Mo

Eduardo Moncada

James A. Morone

Lorenzo Morris

Eric Seth Mosinger

Wynne Walker Moskop

Lama Mourad

Cas Mudde

Russell Muirhead

Ragnhild Louise Muriaas

Tun Myint

Emily Nacol

Eli Naffah

Alessandro Nai

Charles Nathan

National Science

Foundation

Juhem Navarro-Rivera

Dani Nedal

Network for Good

Michael Nest

Dale A. Neuman

Lina Newton

David W. Nickerson

Irfan Nooruddin

Julie L. Novkov

Sally Nuamah

Shayla C. Nunnally

Kazuhiro Obayashi

Cyril Obi

Cleo Marie O'Brien-Udry

Paulina Ochoa Espejo

Thomas K. Ogorzalek

Christopher Ojeda

Alan Okagaki

Ido Oren

Sima R. Osdoby

Diana M. Owen

Timothy Pachirat

Susan M. Park

Sanghee Park

Carole Pateman

Louis Pauly

Joan Paylo

Desiree S. Pedescleaux

Francisco I. Pedraza

Bruce Pencek

Thomas Pepinsky

Sofia A. Perez

Clarisa Perez-Armendariz

Brittany N. Perry

Audrey Perry Martin

Mark A. Peterson

Lance William Pfeifer

Layla Picard

Paula M. Pickering

Dianne M. Pinderhughes
Jessica Piombo

M. Anne Pitcher

Jennifer Pitts

Elizabeth Plantan

David J. Plazek

Mathias Poertner

Heather N. Pool

Alison E. Post

Justin Pottle

G. Bingham Powell Jr.

Antonio Torquilho

Praxedes

Chris Price

Colin Provost

Maria Isabel Puerta

Beryl A. Radin

Nicol C. Rae

Melanie H. Ram

James H. Read

Daniel J. Reagan

Sarah E. Reckhow

Mary Beth Reissen

Andrew S. Reynolds

Molly Reynolds

Guillem Riambau Armet

Nils Ringe

Meg E. Rithmire

Emily Hencken Ritter

Tesalia Rizzo

Kenneth M. Roberts

James C. Roberts

David B. Robertson

Dwayne Robertson

Pearl T. Robinson

Esther Robinson

Ingo Rohlfing

Mark Carl Rom

Laura Roost

Guillermo Rosas

Roger P. Rose

Michelle Rose

Gemma Sala

Stephen G. Salkever

David J. Samuels

Kira Sanbonmatsu

Christopher Michael Sands

Melissa Sands

Aliyu Muhammad Sani

Rodolfo Sarsfield

Deborah Schildkraut

Vivien A. Schmidt

Edward Schneier

Gordon Schochet

Morton Schoolman

Jean Reith Schroedel

Arica N. Schuett
Melissa A. Schwartzberg

Peregrine Schwartz-Shea

Judithanne Scourfield

McLauchlan

Jason Seawright

Tani H. Sebro

Darin Self

Ammar Shamaileh

Henry A. Sheinkopf

Kenneth Sherrill

Michael Dale Siciliano

Heather Silber Mohamed

Dick Simpson

Henry B. Sirgo

Carmen Joseph Sirianni

Steven Rathgeb Smith

Edmond C. Smith Jr

Steven S. Smith

Daniel E. Smith

Lahra Smith

Nicholas Rush Smith

Aaron Smith-Walter

J.H. Snider

Romelia Solano

Daniel Solomon

Sarah Song

Lester Kenyatta Spence

Jae-Jae Spoon

Jeremy Springman

Harvey Starr

Brent S. Steel

Susan C. Stokes

Dietlind Stolle

Alec Stone Sweet

Jonathan R. Strand

Scott Straus

Anton Strezhnev

Alexander Stroh

Tracy B. Strong

Sherrill Stroschein

Philippa Strum

Mary E. Stuckey

Terry Sullivan

Arun R. Swamy

Judith A. Swanson

Jennie Sweet-Cushman

Henrik Syse

Aleksandra Sznajder Lee

Zak Taylor

Jeremy M. Teigen

Andrew Thangasamy

Elizabeth Theiss-Morse

Jessica Thompson

James A. Thurber

Lydia Brashear Tiede

Alvin B. Tillery Jr.

Sue Tolleson-Rinehart 


Peter Trubowitz
Robin L. Turner
Jack Turner III
Mary Evelyn Turner
Lincoln Turner
Ines Valdez
Renee B. Van Vechten
Ashutosh Varshney
Arturo Vega
Mayra Velez-Serrano
Ali Velshi
Jill Mccalla Vickers
Jennifer Nicoll Victor
Kristina M. Victor
Andrea Vilan
Robert Vitalis
Jeffrey J. Volle
Matthias vom Hau
Christopher Austin Wade
Charles E. Walcott
Lee D. Walker
Dror Walter
Julian Wamble
Zhipeng Wang
Myra A. Waterbury

Vesla Mae Weaver

Clayton McLaughlin Webb

Nora Webb Williams Inger Weibust Leonard B. Weinberg Margaret Weir Herbert F. Weisberg Joshua Weitz S. Laurel Weldon Stephanie Werner David Wettergreen William Whisenand Beth Elise Whitaker Donald L. Whitaker II Joseph White Ismail K. White Christopher M. Whitt Ralph R. Widner Jennifer Widner Martha Wilfahrt Sarah Elise Wiliarty Melissa S. Williams Rob Williams Simon Williamson
Graham K. Wilson

Angelia R. Wilson

David M. Wilson

Elizabeth R. Wingrove Jeff Wayne Wismann Jacob P. Wobig

Deva Woodly

Susan L. Woodward

Robinson WoodwardBurns

Kenton W. Worcester Austin L. Wright J. Mark Wrighton Christina Xydias Nicole Yadon Priscilla Yamin Dannagal G. Young Yael Zeira Baobao Zhang Yiqun Zhang J. Nicholas Ziegler Tamas D. Zsitvay Michael P. Zuckert -

\section{6}

I contribute to APSA because I respect it as a well-run professional association that serves students, professionals, and all of society in consequential ways.

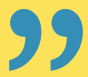

-Beverly A. Cigler, Penn State Distinguished Professor Emerita of Public Policy and Administration

\section{Your will can provide for your family and keep political science thriving for the next generation.}

Make a gift to APSA in your will and join a special group of individuals who, like you, want to have a crucial role in ensuring a bright future for political science scholars, teachers, students, and practitioners.

\section{Remember APSA in your will}

For more information, visit apsanet.org/legacy or email development@apsanet.org 